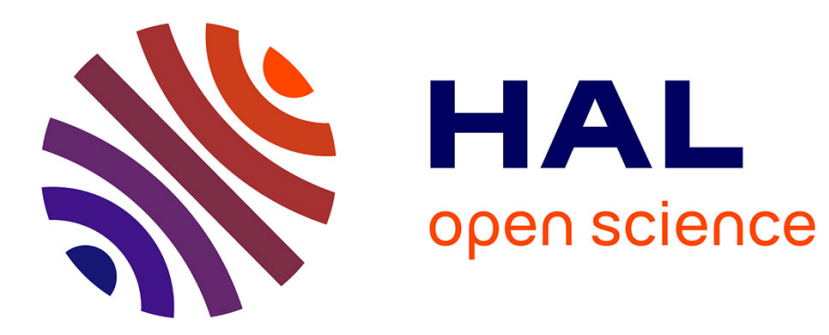

\title{
Les jumelages entre Guerre froide et enjeux locaux
}

William Richier

\section{To cite this version:}

William Richier. Les jumelages entre Guerre froide et enjeux locaux. Sous la direction de Philippe Buton, Olivier Büttner, Michel Hastings. La Guerre froide vue d'en bas, CNRS Editions, p. 131-149, 2014, 9782271078230. halshs-01260097

\section{HAL Id: halshs-01260097 https://shs.hal.science/halshs-01260097}

Submitted on 21 Jan 2016

HAL is a multi-disciplinary open access archive for the deposit and dissemination of scientific research documents, whether they are published or not. The documents may come from teaching and research institutions in France or abroad, or from public or private research centers.
L'archive ouverte pluridisciplinaire HAL, est destinée au dépôt et à la diffusion de documents scientifiques de niveau recherche, publiés ou non, émanant des établissements d'enseignement et de recherche français ou étrangers, des laboratoires publics ou privés. 


\section{Les jumelages entre Guerre froide et enjeux locaux}

William Richier

Les jumelages sont l'expression du fédéralisme européen et plongent leurs racines dans l'entre-deux-guerres. Ils prennent leur essor dans les années 1950, portés par l'espoir d'une Europe solidaire et pacifique, à la croisée de l'internationalisme ouvrier et de l'universalisme catholique. Ils seront toutefois vite emportés par les logiques de Guerre froide : le clivage Est-Ouest sépare une Fédération mondiale des villes jumelées soviétophile d'autres organisations très anticommunistes. Le phénomène des jumelages illustre pleinement ce que fut «la Guerre froide vue d'en bas », puisqu'il fait passer les relations internationales des ambassades aux mairies et aux associations municipales. On peut évoquer une véritable "paradiplomatie » de certains édiles. Les enjeux et usages locaux deviennent déterminants, même si s'esquissent des pratiques globales. L'histoire des jumelages embrasse, en même temps, la question des représentations de l'autre et de leurs traductions idéologiques au niveau local, le souvenir des guerres mondiales ou encore l'implantation des organisations à l'échelle municipale. Il convient d'appréhender également les spécificités de la société politique française, caractérisée par un PCF puissant, suscitant à la fois rejet et fascination et solidement implanté dans ses bastions municipaux comme la ceinture rouge parisienne. L'étude des jumelages bénéficie déjà d'une historiographie de grande qualité ${ }^{1}$ et les articles des correspon-

1. À titre d'exemple on citera: Defrance Corine, «Les jumelages francoallemands. Aspects d'une coopération transnationale ", Vingtième siècle. Revue d'histoire, n ${ }^{\circ} 99$ juillet-septembre 2008, p. 189-201; Belot Robert, « Le jumelage des villes: avatars d'une "bombe de paix" dans la Guerre froide ", in Fleury Antoine, Jilek Lubor (dir.), Une Europe malgré tout : contacts et réseaux culturels et scientifiques entre Européens dans la Guerre froide, Bruxelles, PIE Peter Lang, 2009 ; Vion Antoine, "L'invention de la tradition des jumelages », Revue française de science politique, vol. 53, 2003/4, p. 559-582 ; Bergeret-Cassagne Axelle, Pour une Europe fédérale des collectivités locales. Un demi-siècle de militantisme au sein du Conseil des Communes et des Régions d'Europe (1950-1999), Paris, 
dants départementaux de l'IHTP reflètent la diversité des situations locales. Nous avons ajouté l'étude de trois villes de la région parisienne illustrant à la fois des choix (jumelages avec des municipalités d'Europe de l'Ouest, d'URSS et de RDA) et des objectifs différents de participation populaire. Il convient ainsi de distinguer les jumelages tournés vers l'Ouest de ceux qui franchissent le Rideau de fer.

\section{LES JUMELAGES OUEST-OUEST}

Le maire gaulliste de Nogent-sur-Marne jumelle sa ville avec Siegburg (RFA) en juillet 1964 puis Yverdon (Suisse) l'année suivante ${ }^{2}$. Dans ses Mémoires, il motive sa première décision par le rapprochement francoallemand cher à de Gaulle, et la situation d'une sœur voisine de sa capitale, proche du Rhin et riche de traditions festives. Le choix d'une commune germanique suscite toutefois les réticences des anciens combattants. Quant à la Suisse, il la conçoit comme une "nation symbole de paix et de solidarité » ${ }^{3}$. Son adjoint Jean Maudry affirme que l'apparentement avec Yverdon avait pour fonction de "faire passer la pilule allemande auprès de l'opinion ${ }^{4}$. Les relations sont actives: le jumelage débute par des échanges d'étudiants avec Siegburg dès 1964, et se poursuit par des compétitions sportives, l'envoi de chorales, ou de jeunes Français qui partent travailler dans des usines de la sœur allemande ${ }^{5}$. L'essentiel demeure cependant la participation des villes jumelées à la fête biennale du Petit Vin Blanc, attraction phare de Nogent. Lancée en 1954 à l'initiative de Nungesser, elle s'appuie sur la chanson à succès de Jean Dréjac pour célébrer le mythe des bords de Marne à la Belle Epoque. Elle s'inscrit dans la politique d'aménagement du territoire, et vise à faire de Nogent le centre touristique et festif de la banlieue Est. Une délégation de Siegburg assiste aux réjouissances en 1965 puis en 1967 avec celle d'Yverdon. Nungesser a

L’Harmattan, 2009 ; Chombard-Gaudin Cécile, « Pour une histoire des villes et communes jumelées », Vingtième Siècle. Revue d'histoire, $\mathrm{n}^{\circ}$ 35, 1992, p. 60-66.

2. Une incertitude pèse sur l'année de jumelage avec Yverdon: probablement 1965, peut être 1964.

3. Nungesser Roland, Du petit vin blanc au Palais Bourbon, Paris, Albin Michel, 2003, p. 44.

4. Milza Pierre, Blanc-Chaléard Marie-Claude, Le Nogent des Italiens, Paris, Autrement, 1995, p. 146.

5. Archives municipales de Nogent. Supplément à Gazette Sud, no 466 bis, janvier 1967, p. 45. Carton 3D9/13 Nogent-sur-Marne (supplément annuel au Journal du Val-de-Marne) 1965-1966. 
jumelé sa ville avec des sœurs susceptibles d'amener des touristes. La première est un lieu de détente pour les habitants de Bonn et Cologne, la seconde borde le lac de Neuchâtel. À l'occasion de la cérémonie de réception de la chorale de Siegburg à Nogent en septembre 1968, Michèle Dorman entonne le Petit Vin Blanc après les discours de bienvenue. La chanson prendra au fil des ans un tour quasi officiel dans les relations de jumelage ${ }^{6}$.

Le choix des communes sœurs revêt également une dimension politique. Le 17 juillet 1964, le conseil municipal vote l'appariement avec Siegburg et Yverdon ${ }^{7}$ réalisé sous l'égide de l'Union internationale des maires, organisation née en 1950 à Stuttgart afin de favoriser le rapprochement franco-allemand. Elle avait été pensée par des intellectuels suisses soucieux de promouvoir le modèle helvétique en Europe ${ }^{8}$. Le serment de jumelage avec Siegburg ${ }^{9}$ évoque la « civilisation occidentale » et son « esprit de liberté » qui «s'est d'abord inscrit dans les franchises qu'elles [les anciennes communes] surent conquérir », il se prononce pour " un monde élargi » qui « ne sera vraiment humain que dans la mesure où les hommes vivront libres dans des cités libres », la " fraternité et l'unité européenne », « entreprise de paix et de prospérité ». Outre le comité de jumelage composé d'élus, les associations chrétiennes jouent un rôle essentiel dans les relations avec Yverdon et Siegburg, particulièrement l'Association catholique d'action sociale ou les chorales Vent d'Est et les Moineaux du Val-deMarne. À l'été 1967, ces derniers effectuent une tournée à Aoste, Rome (dans un congrès réunissant 54 nations, consacré par une messe du pape Paul VI), Assise, Yverdon ${ }^{10}$. La chorale de Siegburg, invitée en septembre 1968, est accueillie en allemand par le curé Cordier le dimanche matin et chante une messe avec Vent d'Est ${ }^{11}$. L'idéologie sous-jacente à ces jumelages correspond bien aux valeurs de Konrad Adenauer et de la Loi Fondamentale: l'Europe chrétienne, décentralisée et libre-échangiste contre les totalitarismes. Elle est le fruit du Traité de l’Élysée. Mais elle s'oppose implicitement à la politique du chancelier Erhard et du président de la Commission européenne Walter Hallstein. Roland Nungesser a opté

6. AM de Nogent, "Siegburg et la fleur de tournesol », Le Val-de-Marne, n 19, novembre 1968, p. 5. Carton 407 W.

7. AM de Nogent, délibérations $n^{\circ} 1143$ et 1144, séance du 17 juillet 1964 . Registre des délibérations du conseil municipal du 14 mai 1964 au 10 juin 1966, AM de Nogent, I DI/50.

8. Defrance C., article cité, p. 191.

9. AM de Nogent, Serment de jumelage entre Heinrich, maire de Siegburg, et Roland Nungesser, maire de Nogent-sur-Marne du 20 juin 1964. Carton 407 W. 10. AM de Nogent, "Yverdon-Nogent une amitié s'est nouée », La Gazette du Val-de-Marne, $\mathrm{n}^{\circ}$ 471, septembre 1967, Carton 407W.

11. Ibid., p. 6. 
pour une ville suisse et l'Union internationale des maires (UIM), au lieu du Conseil des communes d'Europe par rejet de la supranationalité.

Au-delà des informations qu'apporte cette étude de cas, les jumelages Ouest-Ouest présentent, à côté de quelques dénominateurs communs, une grande diversité. Les motivations économiques sont essentielles dans les appariements Ouest-Ouest. En 1955, Montpellier se jumelle avec Louisville aux États-Unis sous l'égide du Monde bilingue et prévoit d'y organiser une journée des vins du Languedoc qui ont du mal à se vendre. La ville américaine est de son côté le siège de Brown-Forman, multinationale des alcools. En mai 1957, Montolivet, village du canton de la FertéGaucher, se jumelle avec la commune belge d'Onoz dont le bourgmestre, le comte de Beauffort fut le chef de cabinet de son oncle Charles d'Aspremont, ancien ministre belge de l'Agriculture, à une époque où l'Europe verte se prépare. Évreux est appariée à Rugby (Angleterre) en 1959 et à Rüsselsheim (RFA) en 1961, deux villes au tissu industriel très dense. En 1963, Grenoble s'associe à Innsbruck en vue de préparer les Jeux olympiques d'hiver. Les jumelages à Montolivet et Évreux se constituent sous la houlette du Conseil des Communes d'Europe, très lié à la CEE. Il est à l'origine de la Communauté européenne du crédit communal, créée en 1954 pour faciliter les opérations d'emprunts des collectivités locales en relation avec l'exécutif du Marché Commun ${ }^{12}$. Le CCE touche d'importants financements, notamment du Quai d'Orsay et des Communautés européennes ${ }^{13}$.

Le CCE organise certainement des appariements discrets dans le domaine stratégique. Jean Bareth secrétaire-général du CCE, soutient le jumelage entre Fontainebleau et Constance, respectivement siège du SHAPE et d'une garnison française (1960). Il s'agit du premier jumelage entre une ville de Seine-et-Marne et une commune de RFA. On observe de réelles réticences des municipalités françaises à s'associer à leurs homologues allemandes. La cérémonie de jumelage n'est organisée à Évreux qu'en octobre 1961, après que le maire socialiste de Rüsselsheim eut apporté une aide financière à la ville normande afin de réparer les dégâts causés par un ouragan en juin. Le Limousin, marqué par le souvenir d'Oradour-surGlane, ne connaît aucun jumelage avec une ville allemande avant $1969^{14}$. Corine Defrance montre que les années 1958 et 1963, qui vont de la nais-

12. Bergeret-Cassagne A., op. cit., p. 143.

13. Ibid., p. 69-70.

14. Chombard-Gaudin Cécile , « Des dates et des lieux en Bourgogne Languedoc Roussillon et Limousin », Mappemonde, $\mathrm{n}^{\circ}$ 4, 1995, p. 12. 
sance de la V Vépublique au traité de l’Élysée, marquent l'essor des jumelages franco-allemands ${ }^{15}$.

De manière générale, les motivations politiques qui conduisent aux jumelages Ouest-Ouest sont fondées sur la défense du monde libre, et ses valeurs telles qu'elles étaient notamment portées par les milieux fédéralistes européens de la Résistance socialiste ou chrétienne dont les membres, réfugiés en Suisse, publient en 1944 la Déclaration des Résistances européennes. Ses principaux auteurs sont Ernesto Rossi, Altiero Spinelli et Henri Frenay. Cette mouvance trouve ses sources chez les nonconformistes des années 1930, nébuleuse d'inspiration chrétienne, décentralisatrice, européenne, en quête d'une troisième voie. Ils sont également influencés par des intellectuels anglo-saxons tels que Barbara Wootton et William Beveridge membres du mouvement Federal Union en 1938 ou l'Américain Clarence Streit auteur en 1939 de Union Now ${ }^{16}$. Les socialistes et les chrétiens entretiennent des relations avec les États-Unis, par le biais des exilés ou de l'Office of Strategic Services. En 1942, Allen Dulles se rend en Suisse pour le compte de l'OSS afin de nouer des contacts avec les Résistances européennes et de collecter des renseignements sur le Reich ${ }^{17}$. Au sein du Département d'État, John Foster Dulles, Bullit et Kennan conçoivent le projet d'une fédération européenne, tournée contre l'URSS. Peu entendus par Roosevelt, ils ont cependant l'oreille de Truman. Dulles envisage d'intégrer l'Allemagne dans une Europe fédérale, elle-même incluse dans une union atlantique. Elle serait organisée autour de la Ruhr et du Rhin. Malgré les relais dont disposent les États-Unis dans les milieux fédéralistes européens, ces derniers se méfient de l’Allemagne, restent attachés au maintien de la Grande Alliance et croient dans une Europe «Troisième Force » au-delà des Blocs. L’Union européenne des fédéralistes espère s'implanter dans les pays de l'Est jusqu'à ce que le coup de Prague ne dissipe ses illusions ${ }^{18}$. Le CCE ${ }^{19}$, le Monde bilingue et, de toute évidence, l'UIM partagent l'ambition de développer les échanges, particulièrement dans le domaine commercial, et les projets mutuels de reconstruction entre villes jumelées d'Europe occidentale. Ils bénéficient du soutien officiel du Vatican, de financements patronaux, et puisent leurs

15. Defrance C., article cité, p. 197.

16. Heyde Veronika, De l'esprit de la Résistance jusqu'à l'idée de l'Europe, Bruxelles, Euroclio, 2010, p. 114.

17. Ibid., p. 177 et suivantes.

18. Vayssière Bertrand, «L'Europe aux Européens : les tentatives fédéralistes de s'implanter à l'Est dans l'après-guerre ", in Fleury Antoine et Jilek Lubor (dir.), Une Europe malgré tout: contacts et réseaux culturels et scientifiques entre Européens dans la Guerre froide, Bruxelles, PIE Peter Lang, 2009, p. 205.

19. Conseil des communes d'Europe. 
cadres chez les catholiques de gauche issus de la Résistance, généralement des revues Esprit et Témoignage chrétien. Ces organisations ne dissimulent pas leur commune appartenance à la Communauté atlantique et leur hostilité à l'égard du bloc de l'Est.

Le Monde bilingue est fondé à Paris en août 1951 par Jean-Marie Bressand, Henri Frenay, André Voisin, etc. L'association reçoit notamment le soutien de Daniel-Rops, de Michel Debré, et le parrainage du Président de la République Vincent Auriol ${ }^{20}$. Elle se donne pour but de promouvoir, dès l'école primaire, l'enseignement de l'anglais aux enfants francophones et celui du français aux anglophones en laissant aux jeunes Européens le choix entre l'une et l'autre de ces langues. Ainsi les citoyens du Monde libre auraient tous une seconde langue maternelle qui serait celle de Molière ou de Shakespeare. Le jumelage entre Luchon et la ville anglaise d'Harrogate en 1952 étrenne l'expérience. En 1954, c'est au tour d'Arles et d'York (États-Unis). L'association reçoit le soutien d'Eden, ministre britannique des Affaires étrangères et d'Eisenhower, comme commandant en chef de l'OTAN puis en tant que Président des États-Unis. On constate que le Monde bilingue est très proche des milieux de la défense français et anglo-saxons. Les officiers y sont nombreux, Bressand est lui-même capitaine et son livre met régulièrement en évidence l'intérêt du bilinguisme franco-anglais dans les armées ${ }^{21}$. Après la guerre, les Britanniques ont formé militairement les Français et échangé avec eux des renseignements stratégiques ${ }^{22}$. Il est suggéré dans l'ouvrage de Bressand que l'usage des deux langues s'impose au sein de la Communauté européenne de défense. Cela répondrait au vœu de Paris consistant à faire de la France le troisième pilier de l'OTAN. La volonté de muer Luchon en ville bilingue rappelle le projet d'Union franco-britannique de fusion des souverainetés qu'avait proposé le Royaume-Uni à la France en 1940, et faciliterait le soutien logistique de Londres aux maquis comme cela se pratiquait pendant la guerre. Le Monde bilingue est à l'évidence un de ces réseaux d'actions civiques atlantiques qu'ont déployés les Anglo-Saxons en Europe de l'Ouest au début des années 1950. Ces organisations clandestines devaient préparer la résistance en cas d'occupation soviétique ${ }^{23}$. Les jumelages permettent de drainer des informations à l'échelle internationale ${ }^{24}$.

20. Bressand Jean-Marie, Jean-Marie Bressand présente le monde où l'on s'entend, Paris, le Monde bilingue, 1953, p. 55.

21. Ibid.

22. Sanderson Claire, France, Grande-Bretagne et défense de l'Europe 1945-1958 :

l'impossible alliance ?, Paris, Publications de la Sorbonne, 2003, p. 204-206.

23. Zorgbibe Charles, Histoire de l'OTAN, Bruxelles, Editions Complexe, 2002, p. 121-123.

24. Sanderson C., op. cit., p. 227. 
L’Union internationale des maires que nous avons déjà présentée a parrainé le jumelage de Montbéliard et Ludwigsburg (1950) ${ }^{25}$. Le Conseil des Communes d'Europe est créé à Genève en 1951. Il regroupe alors 26 représentants de collectivités locales originaires de 9 pays et régions: France, RFA, Benelux, Italie, Suisse, Sarre et Danemark ${ }^{26}$. Il est imprégné de la pensée du Suisse Adolf Gasser et d'Emmanuel Mounier, et entend défendre les libertés communales et les droits de la personne contre les totalitarismes. L'influence catholique est si forte qu'il faut attendre l'échec de la CED en 1954 pour que les socialistes y adhèrent. Defferre devient le président de la section française en 1957. Le CCE est très lié à l'Europe supranationale des Six, et promeut les partenariats économiques entre municipalités et régions d'Europe de l'Ouest. Le CCE matérialise les projets des frères Dulles visant à bâtir une Europe organisée autour de la Ruhr et du Rhin et tournée contre l’URSS.

\section{LES JUMELAGES AVEC L’EST : DEUX ÉTUDES DE CAS}

Pantin offre l'exemple d'un jumelage avec l'arrondissement de Dzerjinski, à Moscou, conclu tardivement (1966) et mené sans grand enthousiasme par les maires communistes successifs Jean Lolive (1959-1968) puis Fernand Lainat. Dans un courrier du 4 octobre $1965^{27}$, qu'il adresse vraisemblablement aux membres du conseil municipal, le Premier magistrat annonce que sa commune entretient depuis plusieurs années « des rapports amicaux avec des villes étrangères » et notamment avec Dzerjinski, «rapports qui devraient $[\ldots]$ préparer le jumelage de notre commune avec ces cités ». Il a reçu une invitation des autorités de la ville soviétique et souhaite agréger à la délégation municipale les partis, organisations et personnalités représentatifs de Pantin. Cet œcuménisme correspond à l'esprit de la FMVJ qui entend intégrer, sans exclusive aux jumelages, les diverses sensibilités qui animent les cités, puis y faire participer la population. Les archives de Pantin n'ont pas gardé trace de relations antérieures à 1965, mais il est possible que les deux villes aient établi des liens discrets peu après l'élection d'un maire communiste en 1959, afin de préparer un jumelage en temps

25. Defrance C., article cité, p. 191.

26. Bergeret-Cassagne A., op. cit., p. 7.

27. Archives municipales de Pantin, Lettre de Jean Lolive et V. Etcheverry du 4 octobre 1965. Carton 282W24. 
utile. Une délégation pantinoise arrive à Moscou le 4 novembre $1965^{28}$. Elle est reçue par le Premier vice-Président du Soviet de Moscou, comme si elle représentait la ville de Paris, et retenue jusqu'au 13 novembre, soit deux jours de plus que le programme initial. Ses membres participent même aux manifestations de la Place Rouge pour le $48^{\mathrm{e}}$ anniversaire de la Révolution d'Octobre. Le rang protocolaire qui leur est accordé les oblige, et les incite probablement à voir la capitale soviétique sous un jour très favorable. Moscou leur apparaît comme une municipalité très démocratique comptant un député pour 6000 habitants, des arrondissements dotés chacun d'une assemblée élue, tandis que Paris ne totalise que 90 conseillers municipaux. On a construit 125000 logements de type HLM pour l'année 1965, contre un peu plus de 10000 dans le département de la Seine dont la population est équivalente à celle de Moscou. Le Plan a permis d'édifier des hôpitaux, des crèches, d'étendre un métro plus vaste qu'à Paris et d'améliorer la desserte en eau potable, grâce à une fiscalité plus légère qu'en France. Cela tient à la planification socialiste, élaborée démocratiquement, dans le seul intérêt des populations, à l'opposé du plan capitaliste au service des monopoles. Les thèmes qu'aborde le compte-rendu du voyage sont le quotidien des élus municipaux français. Pantin compte plus de 3000 familles sans logis et mal logés ${ }^{29}$, et l'adoption du V $\mathrm{V}^{\mathrm{e}}$ Plan, en novembre 1964, avait suscité une vague de critiques de la part de la gauche unie derrière la candidature de François Mitterrand. Un contre- plan de Julien Ensemble, préfacé par des syndicalistes FO, CGT, CFDT ${ }^{30}$, dénonçait une planification insuffisamment contraignante et vidée de son contenu par un ministre des Finances donnant la priorité à la lutte contre l'inflation ${ }^{31}$. À un mois des élections présidentielles, le voyage des élus pantinois à Moscou a évidemment pour but de renforcer l'anti-gaullisme au niveau local et d'affaiblir le Président de la République. Le 26 mai 1966, Jean Lolive et Mikaïl Kouznetsov, au nom de Dzerjinski, signent à Pantin une convention de jumelage, au moment où les relations franco-soviétiques s'améliorent.

Mais les Français semblent réticents aux appariements. Les initiatives viennent généralement des Russes, et, en 1970, le comité de jumelage se fixe encore pour objectif d'intégrer toutes les idéologies qui existent dans

28. AM de Pantin, Compte-rendu de la délégation du comité de jumelage municipalité Dzerjinski-Moscou par le maire Jean Lolive, novembre 1965, Carton 282W24.

29. AM de Pantin, entretien du lundi 23 mai. Jumelage Pantin-Dzerjinski. Carton 282W24. Il s'agit très certainement du 23 mai 1966.

30. Ensemble Julien, Le contre-plan, Paris, Le Seuil, 1965. Les syndicalistes sont respectivement Maurice Labi, Pierre Le Brun et René Bonéty.

31. Ibid., p. 26-27. 
la population ${ }^{32}$. L'appariement avec la ville italienne et communiste de Scandicci n'est conclu qu'en 1969, et celui d'une ville de RDA ne le sera jamais malgré les pressions amicales des Allemands. Le premier document concernant l'Allemagne de l'Est à Pantin est une lettre de Fernand Lainat au maire de Francfort-sur-l'Oder du 18 octobre 1969, expliquant pourquoi il n'a pu honorer l'invitation de son homologue ${ }^{33}$ et une note de réunion du 28 octobre $^{34}$ indique "problème : Francfort ou Spremberg ». Les archives du fonds de l'association France-RDA à Bobigny prouvent que des contacts ont été noués dès 1966. En octobre ${ }^{35}, 7$ militants de l'UL-CGT de Pantin sont invités par la FDGB de Spremberg et y rencontrent le maire qui leur remet un courrier invitant son homologue français. La note évoque un éventuel jumelage et en décembre une exposition sur la RDA est organisée à la Bourse du travail de Pantin. Ce type de relations officiellement nouées entre syndicats est caractéristique des relations établies de manières informelles entre les villes communistes françaises et leurs homologues de l'Est, en vue d'un éventuel jumelage susceptible d'être évoqué au moment opportun, pendant une campagne présidentielle par exemple. En juin 1970, les Échanges franco-allemands animent une exposition sur la RDA à la mairie de Pantin ${ }^{36}$. Spremberg semble avoir été oubliée par la suite, et il est seulement question d'une rencontre sportive avec Scandicci et Francfort lors de la réunion du comité de jumelage du 22 janvier $1970^{37}$.

Les archives de la mairie nous renseignent sur le caractère complexe qu'entretiennent les comités de jumelage avec les sociétés d’amitiés en relation avec les pays de l'Est. La FMVJ conçoit les jumelages comme un instrument de culture populaire, à caractère éducatif strictement apolitique. Le Parti cherche à impliquer les sociétés d'amitié dans les relations entre cités. Le maire Fernand Lainat est membre du comité départemental de France-URSS et son camarade et adjoint Marcel Lepron anime un comité local de 27 membres en 1969-1970 ${ }^{38}$. Ce dernier prévoit de se joindre à la

32. AM de Pantin, réunion du comité de jumelage du 9 décembre 1970. Carton $282 \mathrm{~W} 24$.

33. AM de Pantin, lettre de Fernand Lainat, maire de Pantin, au bourgmestre de Francfort-sur-l'Oder, le 18 octobre 1969. Carton 282W25.

34. AM de Pantin CR du voyage de Scandicci, 28 octobre 1969. Carton 282W25. Il s'agit probablement d'une réunion du comité de jumelage.

35. Archives départementales de Bobigny, note d'information Pantin novembre 1966. Carton 38J92, Fonds France-RDA.

36. AD de Bobigny, dépliant «Où va la RDA ? Les Echanges franco-allemands vous invitent ». Carton 38J92, Fonds France-RDA.

37. AM de Pantin, lettre de Fernand Lainat, Président du comité de jumelage, maire de Pantin, 16 janvier 1970 : réunion du comité de jumelage du 22 janvier 1970.

38. AM de Pantin, etat d'organisation France-URSS en Seine-Saint-Denis au 8 septembre 1971. Carton 282W25, Dossier France-URSS. 
municipalité et au comité de jumelage pour accueillir la délégation soviétique attendue pour le 22 juin ${ }^{39}$. En même temps, un courrier du comité départemental de France-URSS au maire de novembre 1970 traduit la colère d'un responsable de l'Association informé par un adjoint d'un prochain séjour à Dzerjinski d'une délégation du comité de jumelage ${ }^{40}$. Il regrette que la municipalité ait fait appel à la société d'amitié pour obtenir les visas et l'ait ignorée pour organiser le voyage.

À ce mariage de raison entre Pantin et Dzerjinski, le jumelage entre Argenteuil et Dessau offre au contraire les qualités exemplaires d'un modèle du genre. Le 12 octobre 1959, Argenteuil a conclu un traité d'amitié avec Dessau. Il s'agit du premier jumelage entre une ville française et une consœur de RDA ${ }^{41}$. Il se caractérise par son dynamisme : Victor Dupouy maire communiste depuis 1935 n'a pas oublié les bombardements qu'a subis sa commune pendant la guerre et s'applique à promouvoir un jumelage modèle sous l'égide de la FMVJ. Selon l'édile, les représentants d'Argenteuil en quête d'une ville étrangère ont rencontré ceux de Dessau lors d'un congrès de l'Union internationale des pouvoirs locaux qui se tenait à Berlin-Ouest en juin 195942. Il est plus probable cependant que des relations discrètes aient été nouées dès $1956^{43}$. Le 18 septembre 1959, le conseil municipal décide à l'unanimité l'adhésion de la commune à la FMVJ et la constitution d'un comité local de jumelage ${ }^{44}$. Le 12 octobre, un traité d'amitié est signé à Dessau, à l'occasion du $\mathrm{X}^{\mathrm{e}}$ anniversaire de la RDA. En

39. AM de Pantin, lettre de Marcel Lepron pour le comité local de France-URSS le 20 juin 1970. Carton 282W25, Dossier France-URSS. La venue de la délégation est finalement ajournée.

40. AM de Pantin, lettre de Pierre Liard, secrétaire général à Fernand Lainat, maire de Pantin, 3 novembre 1969. Carton 282W25, dossier France-URSS. Pierre Liard est membre du comité départemental. Lors de la réunion du comité national d'octobre 1970, il est élu secrétaire national délégué à la promotion des voyages. Comme le suggère le compte-rendu du comité départemental du 13 novembre 1970, il n’occupait pas jusque-là de responsabilité nationale.

41. «Les jumelages entre les villes de France et de RDA se multiplient, mais... », Rencontres franco-allemandes, $\mathrm{n}^{\circ}$ 7, novembre 1960, p. 12.

42. AM d'Argenteuil, « Pourquoi un comité de jumelage ? Une interview de Victor Dupouy, Président du comité de jumelage, Maire d’Argenteuil », La Renaissance d'Argenteuil, 27 février 1960, in 30ème anniversaire du comité de jumelage d’Argenteuil : pour la paix et l'amitié (1959-1989), p. 9. Carton 86W16.

43. AM d'Argenteuil, Lettre du député communiste Léon Feix à Maurice Couve de Murville, ministre des Affaires étrangères, 19 septembre 1966. Carton 61 W5, Léon Feix écrit: «Le vif désir qu’a la Municipalité d’Argenteuil de pouvoir recevoir les représentants de DESSAU s'explique par les liens étroits d'amitié établis depuis 1956 entre les deux villes ».

44. AM d'Argenteuil, extrait du registre des délibérations du conseil municipal le 18 septembre 1959. Carton I4W264. 
novembre, un comité de jumelage de 55 organisations locales est constitué sous la présidence de Victor Dupouy ${ }^{45}$. Il devient définitif le 12 février 1960, et le 29, Victor Dupouy en déclare l'existence au préfet ${ }^{46}$. En novembre, Argenteuil avait prié la FMVJ de lui trouver des sœurs anglaises et italiennes, et entamé des pourparlers avec Noguinsk, ville de la banlieue de Moscou en vue d'un jumelage ${ }^{47}$ qui n'aura jamais lieu. Le $1^{\text {er }}$ mai 1960, Mary Gunns, maire d'Oldbury signe un traité d'amitié à Argenteuil, le 12 juin, c'est au tour de Gemma Enzo, adjoint communiste au maire socialiste d'Alessandria. Dès l'été 1960, Argenteuil envoie des enfants chez ses trois sœurs et en reçoit (à l'exception des Allemands) ${ }^{48}$ tandis qu'en mars 1961, une première délégation de femmes est envoyée à Dessau ${ }^{49}$.

Il est très rare qu'une ville de la FMVJ se jumelle avec une commune de l’Est avant une sœur anglaise. En général, c’est l'inverse qui se produit. À l'évidence, le Parti communiste avait prié Victor Dupouy d'apparier Argenteuil pour le $\mathrm{X}^{\mathrm{e}}$ anniversaire de la RDA. Il a ensuite agi très vite pour nouer d'autres jumelages. Ses choix correspondent pleinement à l'esprit de la FMVJ et de son président, le chanoine Kir. Pour prévenir toute nouvelle guerre capitaliste venant de RFA, il cherche à nouer des contacts avec les Alliés (Grande-Bretagne ${ }^{50}$, URSS) et les Résistants d'hier et de demain (commune italienne gérée par des socialistes, des communistes et des démocrates-chrétiens ${ }^{51}$; Allemagne ouvrière et socialiste), et compte sur la

45. AM d'Argenteuil, traité d'amitié entre les villes d'Argenteuil (France) et Oldbury (Grande-Bretagne) du $1^{\mathrm{er}}$ mai 1960. Carton I4W264.

46. AM d'Argenteuil, Lettre de Victor Dupouy à M. le Préfet du 29 février 1960. Carton I4W264.

47. AM d'Argenteuil, procès-verbal de la réunion du conseil municipal le lundi 9 novembre 1959. Carton I4W264.

48. AM d'Argenteuil, STS 1960 : «La jeunesse d'Argenteuil fait connaissance avec les jeunes Anglais et les jeunes Italiens » par Simone Liénard, adjoint au maire d'Argenteuil. Il s'agit probablement d'un discours. Carton 14W264.

49. AM d'Argenteuil, «Une première délégation de travailleurs d'Argenteuil se renseigne à Dessau sur les organisations sociales, de santé et scolaire », compterendu de 1961. Carton 3D23-27.

50. Lire à cet égard : «Depuis le $1^{\text {er }}$ mai Argenteuil est officiellement jumelée à Oldbury », La Renaissance d'Argenteuil, nº 783, 7 mai 1960, AM d'Argenteuil, carton 3D23-27. L'article est sous-titré « Entente cordiale ».

51. « Nos amis Italiens sont là », La Renaissance d'Argenteuil. AM d'Argenteuil, carton 3D23-27, date du journal non indiquée, probablement le $n^{\circ} 788$ du 11 juin 1960. Parmi les délégués italiens qui se sont rendus à Argenteuil pour signer le traité d'amitié figure l'élu communiste William Valsesia, ancien FTP français. 
participation des masses ${ }^{52}$. C'est la raison pour laquelle un comité de jumelage, tel celui d'Argenteuil, doit être représentatif.

En janvier 1961, ce dernier comprend 54 organisations ${ }^{53}$ parmi lesquelles l'Association des anciens combattants volontaires la Résistance, Orgemont sport, Société d'horticulture, la JOC, la symphonie d'Argenteuil, l'Action catholique générale féminine, le syndicat des Communaux, le PCF, le PSA, l'UGS, l'Union touristique les Amis de la nature, l'aéroclub, les déportés du travail et réfractaires, l'Amicale des Bretons d'Argenteuil, les Scouts de France, la Croix-Rouge, Estudiantina, le Billard Club, France-URSS, le SNI, les associations de parents d'élèves, les unions locales CGT et CFTC, l'ARAC, I'UFF, l'Union des vieux travailleurs, les Amitiés franco-chinoises, la Fédération nationale des déportés, internés, résistants, patriotes, le Mouvement de la paix, l’Union progressiste, les syndicats des architectes, etc. Cet œcuménisme justifie à la fois la représentativité et l'apolitisme du comité de jumelage, puisque l'on trouve aux côtés d'associations très diverses, le PCF et la gauche non communiste, notamment chrétienne. En pratique, la municipalité et ses nombreuses organisations satellites (Mouvement pour la Paix, UFF, ARAC, CGT, etc.) garantissent au Parti communiste le contrôle du comité de jumelage. Plus de 900 Argenteuillais se rendent à Dessau entre octobre 1959 et novembre $1970^{54}$ parmi lesquels des élus, des féministes, des chrétiens, des socialistes, d'anciens déportés, des joueurs de football, des enseignants, des syndicalistes, etc. En 1969, à l'occasion du $\mathrm{X}^{\mathrm{e}}$ anniversaire du jumelage et des vingt ans de la RDA, Thea Rauschild, maire de Dessau, et Simone Liénard, adjointe et secrétaire du comité de jumelage d'Argenteuil, sont décorées par la « Ligue de l'Amitié entre les peuples de RDA » ${ }^{55}$.

Le comité de jumelage d'Argenteuil est extrêmement actif, y compris dans les relations commerciales avec Dessau. Ainsi sa commission économique se donne pour but de faire connaître aux Argenteuillais les produits

52. Comme le dit Félix Kir dans son appel aux maires du monde au printemps 1959 « Le jumelage a pour but de mettre en relation les classes populaires. Pratiquement, et l'histoire est là pour le confirmer, ce sont les masses populaires qui sont capables de maintenir la paix » : in Le bureau de la Fédération mondiale des villes jumelées et les membres de la commission permanente vous invitent à adhérer à la Fédération mondiale des villes jumelées. AM d'Argenteuil, carton 14W264.

53. « Chronique des villes jumelées », Rencontres franco-allemandes, $\mathrm{n}^{\circ} 8$ janvier, 1961, p. 16.

54. AM d'Argenteuil, lettre de Roger Bahier, secrétaire du comité de jumelage, 7 novembre 1970. Carton 61W5.

55. AM d'Argenteuil, « Haute distinction pour le maire ». Carton 3D23-27. 
fabriqués dans la ville sœur ${ }^{56}$. En 1969, il est prévu de construire dans la commune un hall d'exposition de machines fabriquées en RDA ${ }^{57}$. De surcroît, cette dernière se sert des jumelages pour importer des technologies, des savoir-faire, voire des marchandises. En août 1968, 9 apprentis du bâtiment, encadrés par le directeur d'un collège d'enseignement technique, sont invités à Dessau ${ }^{58}$ pour construire un HLM lors d'un séjour travailloisir ${ }^{59}$. À la fin des années 1960, des urbanistes d'Argenteuil font des exposés dans la commune sœur ${ }^{60}$. Cette dernière demande que les Français lui envoient une délégation de chefs d'entreprises pour septembre $1970^{61}$. À l'inverse, en 1969, Argenteuil prie son amie de recevoir des artisans en difficultés plutôt que des commerçants ${ }^{62}$. La RDA fournit un marché captif et peu exigeant qui pourrait sauver des PME de la faillite.

Le traité d'amitié Argenteuil-Dessau du 12 octobre 1959 prévoit la constitution d'un comité d'amitié dans chaque commune, placé sous la direction du maire et chargé de collaborer notamment avec les associations culturelles avec l'étranger ${ }^{63}$. Cette clause, qui n'apparaît pas dans les pactes conclus avec Oldbury et Alessandria, est destinée aux Échanges franco-allemands nés en avril 1958. Mais ces derniers ne figurent pas sur la liste fondatrice du comité de jumelage lors de la réunion du 9 novembre 1959 à laquelle France-URSS s'était rendue ${ }^{64}$. En janvier 1961, cette clause n'est toujours pas mentionnée ${ }^{65}$. On trouve très peu de traces d'un

56. « Chronique des villes jumelées », Rencontres franco-allemandes, $\mathrm{n}^{\circ} 8$, janvier 1961, p. 20.

57. AM d'Argenteuil Lettre de Simone Liénard à Gérard Auerswald du 11 juin 1969. Carton 14W264.

58. AM d'Argenteuil, carton 14W26. II est entendu qu'ils paient le déplacement. Voir lettre de Simone Liénard du 30 avril 1968.

59. AM d'Argenteuil, Ibid. Voir aussi la lettre de Simone Liénard du 21 mars 1968. Carton 14W264. Le directeur est M. Despierre.

60. AM d'Argenteuil, lettre de Simone Liénard à Thea Rauschild, maire de Dessau, 29 mars 1968, et lettre de Victor Dupouy, maire d'Argenteuil, à Thea Rauschild, maire de Dessau, 7 août 1969, carton 14W264.

61. AM d'Argenteuil Lettre de Roger Bahier, secrétaire du comité de jumelage, à Thea Rauschild, maire de Dessau, 2 juin 1970. Carton 14W264.

62. Lettre de Simone Liénard à Thea Rauschild, maire de Dessau, 18 janvier 1969. AM d'Argenteuil, carton 14W264,

63. Traité d'amitié entre les villes de Dessau (République Démocratique Allemande) et d'Argenteuil (France), 12 octobre 1959. AM d'Argenteuil, carton 14W264.

64. AM d'Argenteuil, procès-verbal de la réunion du 9 novembre 1959, salle de la bibliothèque, sous la présidence de M.Victor Dupouy, maire d'Argenteuil, carton 14W264.

65. «Chronique des villes jumelées », Rencontres franco-allemandes, $\mathrm{n}^{\circ} 8$, janvier 1961, p. 16. 
comité local, hormis dans l'organisation d'une exposition en $1969^{66}$, et dans un document datant peut-être du début des années $1960^{67}$ : le bureau directeur s'est réuni le 24 octobre "afin de discuter des incidents créés dans le comité de jumelage » par l'exposition et les conférences sur la $\mathrm{RDA}$ réalisées par les EFA «que le compte-rendu du journal $L a$ Renaissance attribuait au patronage du comité de jumelage ", car « aucune manifestation politique, de quelque origine soit-elle n'est admise au Jumelage qui voulant avant tout autre chose, comme l'a justement fait remarquer Monsieur le Maire, réaliser cette coexistence pacifique si prônée !, se doit de faire régner déjà à notre base cette entente sans laquelle il ne peut y avoir de travail efficace». Ainsi « les organisations qui peuvent prêter à confusion avec le jumelage soit les Échanges franco-allemands ou France-URSS prendront grand soin de se réserver l'exclusivité de leurs activités ». À l'évidence, Victor Dupouy a délibérément écarté les EFA du comité de jumelage afin d'éviter les conflits en son sein et de respecter au mieux l'apolitisme, cher à la FMVJ, quitte à négliger une clause du traité d'amitié probablement imposée par Dessau et le PCF.

\section{LES JUMELAGES AVEC L’EST : UNE DIPLOMATIE PAR LE BAS}

L'échec de la CED en août 1954 conduit les Occidentaux à réarmer la RFA, et à l'intégrer dans l'OTAN en mai 1955. Cela inquiète d'autant plus Moscou que Bonn n'a pas reconnu ses frontières orientales, et en juillet Khrouchtchev proclame la doctrine des deux États allemands. Les principaux enjeux de l'évolution du Monde bilingue sont ainsi scellés. En 1955, l'association accueille des progressistes (Pierre Cot), des gaullistes de gauche (le général Billotte, René Capitant) et défend le principe d'un équilibre entre les pays anglo-saxons et le bloc de l'Est. En avril 1957 elle devient la Fédération mondiale des villes jumelées et, en septembre 1959, le chanoine Kir signe, sous ses auspices, un pacte d'amitié. Elle éveille l'hostilité du CCE et de la SFIO ${ }^{68}$. Khrouchtchev est conscient de la fragilité du glacis, et s'inquiète que la RFA, réarmée et redevenue la première

66. AM d'Argenteuil, « Une place d'un nouveau quartier d'Argenteuil sera la place Dessau », document manuscrit, ni daté ni signé. Carton 3D23-27.

67. AM d'Argenteuil. Extrait du procès-verbal de la réunion du bureau directeur, comité de jumelage d'Argenteuil, carton I4W264. Malheureusement non daté. Les archives des journaux La Renaissance ou L'Eclair permettraient d'établir une date précise à cet événement survenu en octobre.

68. Voir Vion A., article cité. 
puissance industrielle du continent, reconstitue son Hinterland en renouant des relations commerciales avec des démocraties populaires, lasses de la férule soviétique. Le frêle régime d'Ulbricht, pas même reconnu par les Occidentaux, et qui perd massivement sa main-d'œuvre qualifiée via Berlin, est le plus vulnérable. Moscou entend obtenir la reconnaissance de la RDA, l'instauration d'organismes pan-allemands, la neutralisation de Berlin et la démilitarisation de l'Europe centrale, y compris de l'Allemagne, ce qui aurait pour effet de mettre Bonn et Paris à la merci de l'armée Rouge. Dès 1955, l’URSS entreprend de coordonner les plans des démocraties populaires et de les spécialiser afin de les préserver d'une relation de dépendance économique vis-à-vis de la RFA et des États-Unis. Parallèlement, Moscou cherche à développer les échanges avec l'Europe de l'Ouest et à importer des technologies occidentales pour assurer le succès du Plan septennal (1959-1965). Cela suppose à la fois de multiplier les partenaires commerciaux pour réduire la part de la RFA qui est en 1959 la principale exportatrice ouest-européenne en direction de l'Est.

Les projets du Kremlin s'articulent autour d'une double stratégie : le chantage nucléaire et le discours lénifiant de la coexistence pacifique dont les jumelages sont l'expression. Les sociétés d'amitié sous influence communiste en sont l'adjuvant nécessaire, et si France-URSS ou FranceHongrie est ancienne, la création des Échanges franco-allemands en avril 1958 atteste la primauté de la question berlinoise dans la politique soviétique. Comme l'indique une note interne au PCF: «Il faut rappeler que c'est en 1958 que la direction du Parti a décidé la création des EFA et a demandé aux camarades à qui elle a confié cette responsabilité, d'œuvrer afin de placer cette organisation, immédiatement après France-URSS, compte tenu de l'importance et de l'urgence du problème ${ }^{69}$. Les comités de jumelage et les sociétés d'amitié cherchent à attirer des milieux sociaux et des sensibilités politiques non communistes, notamment les anciens résistants, les pacifistes, les progressistes, les partisans des États-Unis d'Europe et les nostalgiques de la Grande Alliance. La gauche dissidente, mendésiste ou chrétienne est particulièrement visée. La présence du Doyen Châtelet à la tête des EFA, ou de Charles Hernu au sein du Comité national n'est pas fortuite. Lors du $6^{\mathrm{e}}$ congrès des villes jumelées à Paris en septembre 1967, le catholique La Pira, ancien maire de Florence, engage toutes les villes du monde à coopérer au sein de la FMVJ contre « les im-

69. Note au secrétariat fédéral sur l'activité dans la Fédération concernant l'amitié franco-allemande, Archives départementales de Seine-Saint-Denis, fonds FranceRDA 38J2. 
périalismes, les racismes et fascismes sans cesse renaissants " ${ }^{70}$, s'inscrivant dans la politique extérieure du Kremlin. Les sociétés d'amitié et les jumelages reçoivent le soutien de gaullistes de gauche, tels que le général Billotte, cofondateur de l'UDT, membre de la FMVJ, ministre des DOM-TOM de 1966 à 1968, et régulièrement sollicité pour demander des visas. En pratique toutefois, les jumelages et sociétés d'amitié semblent attirer surtout des opposants au Général ${ }^{71}$.

La FMVJ et les sociétés d'amitié parviennent à toucher des secteurs traditionnellement très éloignés du PCF tels que les entrepreneurs et des élus de droite. Le radical Emile Roche, Président du Conseil économique et social, proche des milieux d'affaires se rend à la première réunion du Sénat du conseil économique et culturel des villes jumelées (filiale de la FMVJ, condamnée comme telle par le Conseil de l’Europe en 1961), pour y défendre le principe du libre-échange avec la RDA ${ }^{72}$. La FMVJ et les sociétés d'amitié permettent à Moscou d'étendre son réseau d'influence à toute l'Europe de l'Ouest, et particulièrement à des pays où l'on craint la reconstitution d'une grande Allemagne dans ses frontières de 1937. Comme l'indique une note interne du PCF à propos d'une délégation à Eisenhüttenstadt de syndicalistes de ses villes sœurs Willenhall et Drancy : " que des militants du Labour Party viennent en RDA grâce à la charnière française ne peut qu'aider à faire lever le barrage que les sociauxdémocrates de RFA font peser sur la $\mathrm{RDA}^{73}$ ». L'Allemagne de l'Est est présentée aux entrepreneurs et aux centristes comme une démocratie parlementaire ${ }^{74}$, un double de la RFA acquis au libre-échange comme l'est en principe l'Allemagne de Erhard. La foire de Leipzig est systématiquement mise en valeur. En septembre 1964, elle est organisée sous le thème: « Pour un commerce étendu au monde entier et pour la coopération économique ». Elle semble compléter le Kennedy Round qui s'est ouvert en mai $^{75}$. Il n'est guère étonnant, dès lors, que la « cinquième puissance industrielle d'Europe ${ }^{76}$, puisse vivre également son «miracle écono-

70. «La Renaissance » du 16 septembre 1967, dossier « Petrodvoretz - Petrerhof » Archives municipales du Blanc-Mesnil.

71. Le nom de François Mitterrand apparaît pour la première fois dans le numéro d'octobre 1962 des Rencontres franco-allemandes.

72. Rencontres franco-allemandes, $\mathrm{n}^{\circ}$ 8, janvier 1961.

73. Note d'André Renard au secrétariat fédéral le 8 juillet 1965, AD de SeineSaint-Denis, fonds France- RDA 38J2.

74. Bories Emile, «Les partis en RDA », Rencontres franco-allemandes, $\mathrm{n}^{\circ} 7$, novembre 1960, p. 13.

75. Rencontres franco-allemandes, $\mathrm{n}^{\circ}$ 29, octobre 1964, p. 11.

76. Rencontres franco-allemandes, $\mathrm{n}^{\circ}$ 5, juin 1960, p. 2. 
mique ${ }^{77}$. Plus globalement, l'image des pays de l'Est s'adapte au public visé : lors du séjour des élus pantinois à Moscou en novembre 1965, celleci devient l'éden des édiles de la région parisienne. Si les jumelages avec l'Est entrent bien dans le cadre de la diplomatie soviétique, ils font également des municipalités les acteurs locaux d'une diplomatie par le bas. Ainsi la municipalité jumelée remplit la fonction essentielle de support logistique. Elle soutient des organisations satellites du PCF, associe les non-communistes, et sert de relais à l'implantation d'autres types de jumelages avec l'Est: écoles, universités, associations de loisirs, de culture, entreprises, notamment grâce aux différentes commissions qui composent le comité de jumelage $(\mathrm{CJ})^{78}$. Sont privilégiées les relations avec les milieux culturels, enseignants (afin de toucher les milieux socialistes ${ }^{79}$ et les parents d'élèves), les industriels, commerçants et artisans. Les jumelages sont généralement pensés à l'échelle d'un département, voire d'une conurbation dominée par une métropole ${ }^{80}$. Les communes de banlieues dirigées par le PCF se jumellent avec des villes de l'Est, tandis qu'au même moment les sociétés d'amitié préparent un appariement dans la métropole (Paris-Berlin, Lyon-Leipzig, Lille-Erfurt). À terme, il s'agit de jumeler des départements français à leurs homologues de l'Est. En région parisienne, les EFA cherchent systématiquement à marier les gares avec celles de RDA. Les obstacles officiels aux jumelages avec l'Est sont nombreux, procédant à la fois de l'État et de la FMVJ. L'Allemagne de l'Est n'est pas reconnue par la France avant 1973 et le gouvernement se méfie de l'URSS. Les visas sont refusés aux Allemands de l'Est invités par les villes jumelées, et les maires de Glashütte et Eisenhüttenstadt se rendent en SeineSaint-Denis pour la première fois en juillet $1968^{81}$. Le maire communiste de Malakoff, Léo Figuères, adresse au Préfet une demande de jumelage avec un quartier de Moscou en mai 1965 que l'intéressé ne transmet au

77. Débat organisé par le comité EFA de Drancy et animé par Jacques Farkas en novembre 1969. Voir l'affiche « En l’honneur du 20e anniversaire de la République démocratique allemande ». AM Drancy, carton « Eisenhüttenstadt 1968 à 1970 ». 78. Jeunesse et enfance, sports, affaires culturelles, enseignement, syndicats et jumelages, entreprises à Saint-Denis, voir également le comité de jumelage de Saint-Étienne-du-Rouvray avec Novaïa Kakhovska en URSS, in Gomart Thomas, Double détente: les relations franco-soviétiques de 1958 à 1964, Paris, Publications de La Sorbonne, 2003 p. 138.

79. AD de Seine-Saint-Denis. Note du 21 décembre 1967 sur la réunion du 19 décembre des militants des comités de jumelage et des responsables des comités EFA de Seine-Saint-Denis par André Renard. Fonds France-RDA 38J2.

80. Particulièrement la région parisienne, le Rhône et Nord-Pas-de-Calais dans les contacts avec la RDA.

81. AD de Seine-Saint-Denis, La Voix de l'Est, 24 juillet (?) 1968, fonds FranceRDA 38J87. 
ministère de l'Intérieur qu'en août 1966. En 1968, le quai d'Orsay n’a toujours pas donné sa réponse. Le Blanc-Mesnil, qui a conclu un jumelage avec Petrodvorets (URSS) en 1961, ne peut accueillir de délégation de sa ville sœur avant septembre 1967 faute de visas. La Charte de la FMVJ interdit l'utilisation des jumelages à des fins partisanes ou politiques. C'est pour garantir la neutralité théorique de ces derniers que le PCF compte sur les sociétés d'amitié. Celles-ci, qui ont vocation à attirer les masses, doivent être représentées au sein des comités de jumelage, et s'appuyer sur ses réalisations concrètes pour agir politiquement. En d'autres termes, le comité de jumelage est cantonné à des tâches techniques, et fortement contrôlé par le PCF. Mais ce schéma est difficile à mettre en œuvre. À Pantin et Argenteuil, l'association France-URSS et les EFA sont respectivement tenus à l'écart par les municipalités communistes. En 1969, sur les 11 villes jumelées avec la RDA en Seine-Saint-Denis, deux comités EFA naissent dans l'année (Bagnolet et Montfermeil ${ }^{82}$ ) et quatre communes s'en dispensent encore (Bobigny, Dugny, Saint-Denis, Villetaneuse ${ }^{83}$ ).

Les sociétés d'amitié sont jugées politiquement très connotées. Leur rôle devient décisif lorsque la commune n'est pas appariée avec un pays de l'Est. Le PCF crée alors une association locale d'amitié qui reçoit pour mission de préparer un jumelage et se conduit comme un comité de jumelage. À Amiens, le comité d'amitié avec Görlitz est créé en 1963 et animé par Roger Kiintz, responsable local du Mouvement pour la Paix, de France-URSS et candidat communiste aux élections municipales de 1965. Le comité local d'Amiens, jumelé avec un comité de Görlitz, envoie 15 enfants dans la ville sœur en 1963, et mène une activité intense. Rencontres franco-allemandes présente cette relation comme un véritable jumelage ${ }^{84}$ alors que celui-ci n’est voté par le conseil municipal que le 12 juin 1971, juste après la victoire du communiste René Lamps aux élections de mars ${ }^{85}$. L'histoire des sociétés d'amitié en l'absence d'un comité de jumelage mériterait une étude approfondie.

L'organisation des jumelages avec l'Est au niveau local est donc une tâche ingrate. Dans le cas de la RDA, l'État n'accorde aucune subvention

82. Note au secrétariat fédéral sur les perspectives concernant le futur comité départemental des Echanges franco-allemands par André Renard le 20 mars 1969, AD de Seine-Saint-Denis, fonds France-RDA 38J Dossier 2 courriers.

83. AD de Seine-Saint-Denis. Note à Jacques Denis sur les relations EFA-comités de jumelage par André Renard le 18 avril 1969, fonds France-RDA 38J Dossier 2 courriers.

84. Rencontres franco-allemandes, $\mathrm{n}^{\circ}$ 31, décembre 1964, p. 2.

85. Cahon Julien, « René Lamps un instituteur et un élu communiste à l'épreuve du pouvoir local et de la gestion municipale », http://chs .université-parisl.fr. 
aux municipalités. Les relations avec le pays d'Ulbricht représentent une surcharge de travail, un coût important, et il n'est pas facile de convaincre les Français des vertus pacifistes de la RDA. Les relations entre les Français et les villes jumelées est-allemandes ne sont en effet pas toujours aisées. La culture démocratique des premiers se heurte aux valeurs et pratiques d'un État totalitaire. Les Allemands de l'Est n'arrivent pas quant à eux à comprendre que les communes françaises sont politiquement contraintes par les statuts de la FMVJ, et exigent d'elles qu'elles copient sans discernement la propagande soviétique. Le contrat d'amitié signé entre Gera et Saint-Denis le 9 mars 1963 évoque le combat des citoyens de cette dernière contre "la dictature de De Gaulle ». Une note manuscrite commente : « vœux pieux et inacceptable pour la FMVJ » ${ }^{86}$. Les communes de RDA sont très réticentes devant des jumelages qui leur sont imposés par l'État. Rüdersdorf, partenaire de Pierrefitte, tarde systématiquement à répondre aux courriers de sa sœur française ou omet de signer le contrat d'amitié de l'année 1968-196987. Il avait fallu trois ans pour qu'Eisenhüttenstadt finisse par accepter son appariement. Les séjours des Français en RDA sont parfois malaisés. Les jeunes gens qui se rendent à Potsdam en 1969, rechignent à la discipline militaire du camp, se plaignent de l'éducation politique qu'ils subissent et se voient opposer des annulations ou des refus lorsqu'ils demandent à visiter une boulangerie ${ }^{88}$. Les jumelages participent d'un idéal européen gagé sur la paix, la reconstruction et la coopération afin de rompre avec les désastres des deux guerres. Ce projet d'amitié entre les peuples subit les déchirures de la Guerre froide, et la nature des appariements s'avère très différente, selon qu'ils émanent d’États démocratiques ou totalitaires.

86. AD de Seine-Saint-Denis. « Contrat d’amitié entre la ville de Gera et la ville de Saint-Denis », 9 mars 1963, fonds France-RDA 38J98 Saint-Denis-Gera.

87. AD de Seine-Saint-Denis. Lettre de Roger Fréville à André Renard le 19 mars 1969, fonds France-RDA 38J92 Pierrefitte.

88. AD de Seine-Saint-Denis. Compte-rendu anonyme du voyage à Potsdam en août 1969, p. 3. Il s'agit d'un séjour vacances-loisirs de jeunes gens d’Aubervilliers, fonds France-RDA 38J86 Aubervilliers. 